\title{
The Duffing Oscillator Equation and Its Applications in Physics
}

\author{
Alvaro Humberto Salas Salas $\mathbb{D}^{1},{ }^{1}$ Jairo Ernesto Castillo Hernández $\mathbb{D}{ }^{2}$ \\ and Lorenzo Julio Martínez Hernández $\mathbb{D D}^{3,4}$ \\ ${ }^{1}$ Universidad Nacional de Colombia, Department of Mathematics and Statistics, Fizmako Research Group, Bogotá, Colombia \\ ${ }^{2}$ Universidad Distrital Francisco José de Caldas, Fizmako Research Group, Bogotá, Colombia \\ ${ }^{3}$ Universidad Nacional de Colombia-Manizales-Caldas, Department of Mathematics and Statistics, Caldas, Colombia \\ ${ }^{4}$ Universidad de Caldas, Department of Mathematics and StatisticsManizales, Caldas, Colombia
}

Correspondence should be addressed to Alvaro Humberto Salas Salas; ahsalass@unal.edu.co

Received 21 March 2021; Accepted 23 August 2021; Published 30 November 2021

Academic Editor: Maria L. Gandarias

Copyright (C) 2021 Alvaro Humberto Salas Salas et al. This is an open access article distributed under the Creative Commons Attribution License, which permits unrestricted use, distribution, and reproduction in any medium, provided the original work is properly cited.

In this paper, we solve the Duffing equation for given initial conditions. We introduce the concept of the discriminant for the Duffing equation and we solve it in three cases depending on sign of the discriminant. We also show the way the Duffing equation is applied in soliton theory.

\section{Introduction}

The nonlinear equation describing an oscillator with a cubic nonlinearity is called the Duffing equation. Duffing [1], a German engineer, wrote a comprehensive book about this in 1918. Since then there has been a tremendous amount of work done on this equation, including the development of solution methods (both analytical and numerical) and the use of these methods to investigate the dynamic behavior of physical systems that are described by the various forms of the Duffing equation. Because of its apparent and enigmatic simplicity, and because so much is now known about the Duffing equation, it is used by many researchers as an approximate model of many physical systems or as a convenient mathematical model to investigate new solution methods [2-7]. This equation exhibits an enormous range of well-known behavior in nonlinear dynamical systems and is used by many educators and researchers to illustrate such behavior. Since the 1970s, it has become really popular with researchers into chaos, as it is possibly one of the simplest equations that describes chaotic behavior of a system. This equation is also useful in the study of soliton solutions to important physics models such as $\mathrm{KdV}$ equation, $\mathrm{mKdV}$ equation, sine-Gordon equation, Klein-Gordon equation, nonlinear Schrodinger equation, and shallow water wave equation [8-18].

\section{Undamped and Unforced Duffing Equation}

Let $p, q, u_{0}$, and $\dot{u}_{0}$ be real numbers. The general solution to the undamped and unforced Duffing equation $u^{\prime \prime}(t)+p u(t)+q u^{3}(t)=0$ may be expressed in terms of any of the twelve Jacobian elliptic functions, as shown in Table 1.

In this section, we will solve the initial value problem

The number

$$
u^{\prime \prime}(t)+p u(t)+q u^{3}(t)=0, \quad u(0)=u_{0}, u^{\prime}(0)=\dot{u}_{0},\left(u_{0}^{2}+\dot{u}_{0}^{2}\right) q \neq 0 .
$$

$$
\Delta=\left(p+q u_{0}^{2}\right)^{2}+2 q \dot{u}_{0}^{2}
$$


TABle 1: Equivalent solutions to a Duffing equation.

$$
\begin{aligned}
& u(t)=c_{1} \operatorname{cn}\left(\sqrt{q c_{1}^{2}+p} t+c_{2} \mid\left(q c_{1}^{2} / 2\left(q c_{1}^{2}+p\right)\right)\right) \\
& u(t)=c_{1} \operatorname{sn}\left(\left(\left(\sqrt{q c_{1}^{2}+2 p}\right) / \sqrt{2}\right) t+c_{2} \mid-\left(q c_{1}^{2} /\left(q c_{1}^{2}+2 p\right)\right)\right) \\
& u(t)=c_{1} \operatorname{dn}\left(\left(\sqrt{q c_{1}^{2}} / \sqrt{2}\right) t+c_{2} \mid\left(2\left(q c_{1}^{2}+p\right) / q c_{1}^{2}\right)\right) \\
& u(t)=c_{1} \operatorname{sc}\left(\left(\left(\sqrt{q c_{1}^{2}-2 p}\right) / \sqrt{2}\right) t+c_{2} \mid\left(2\left(p-q c_{1}^{2}\right) /\left(2 p-q c_{1}^{2}\right)\right)\right) \\
& u(t)=c_{1} \operatorname{sd}\left(\sqrt{q c_{1}^{2}-\sqrt{q^{2} c_{1}^{4}+p^{2}}} t+c_{2} \mid\left(\left(q c_{1}^{2}+p+\sqrt{q^{2} c_{1}^{4}+p^{2}}\right) / 2 p\right)\right) \\
& u(t)=c_{1} \operatorname{dc}\left(\left(\sqrt{-q c_{1}^{2}} / \sqrt{2}\right) t+c_{2} \mid\left(-q c_{1}^{2}-2 p\right) / q c_{1}^{2}\right)
\end{aligned}
$$

is called the discriminant for problem (1).

2.1. First Case: $\Delta>0$. In the case, when $\dot{u}_{0}=0$, we get $\Delta=$ $\left(p+q u_{0}^{2}\right)^{2}$ so that $p+q u_{0}^{2} \neq 0$ and the problem reduces to

$$
u^{\prime \prime}(t)+p u(t)+q u^{3}(t)=0, \quad \text { subject to } u(0)=u_{0}, u^{\prime}(0)=0 \text {. }
$$

Its solution is given by

$$
u(t)=u_{0} \mathrm{cn}\left(\sqrt{p+q u_{0}^{2}} t, \frac{q u_{0}^{2}}{2\left(p+q u_{0}^{2}\right)}\right) .
$$

Let $\dot{u}_{0} \neq 0$. First of all, observe that if $u=u(t)$ is a solution to the ode $u^{\prime \prime}(t)+p u(t)+q u^{3}(t)=0$, then $U(t)=$ $u(t+C)$ is also a solution for any constant $C$. Secondly, let $y(t)=c_{1} \operatorname{cn}(\sqrt{\omega}, m)\left(c_{1}=\right.$ a nonzero constant $)$ be the Jacobi elliptic function $c n$ with modulus $m$ and parameter $k$ defined by $k^{2}=m$. We have

$$
y^{\prime \prime}(t)+(1-2 m) \omega y(t)+\frac{2 m \omega}{c_{1}^{2}} y^{3}(t)=0, \quad \text { for any } t, \text { any } c_{1} \neq 0
$$

Therefore, comparing (1) and (5) gives

$$
\begin{gathered}
m=\frac{q c_{1}^{2}}{2\left(p+q c_{1}^{2}\right)}, \\
\omega=p+q c_{1}^{2},
\end{gathered}
$$

and we conclude that the analytic function

$$
u=u(t)=c_{1} \mathrm{cn}\left(\sqrt{p+q c_{1}^{2}} t+c_{2}, \frac{q c_{1}^{2}}{2\left(p+q c_{1}^{2}\right)}\right), \quad c_{1} \neq 0,
$$

is the general solution to the Duffing equation $u^{\prime \prime}(t)+p u(t)+q u^{3}(t)=0$ for arbitrary constants $c_{1}$ and $c_{2}$. The values of these constants are determined from the initial conditions $u(0)=u_{0}$ and $u^{\prime}(0)=\dot{u}_{0}$.

We have

$$
\begin{aligned}
\operatorname{cn}\left(\mathrm{cn}^{-1}\left(u_{0}, m\right), m\right) & =u_{0}, \quad \text { so that } c_{2}=\mathrm{cn}^{-1}\left(u_{0}, m\right), \\
c_{2} & =\mathrm{cn}^{-1}\left(u_{0}, \frac{q c_{1}^{2}}{2\left(p+q c_{1}^{2}\right)}\right), \\
\operatorname{cn}\left(c_{2}, \frac{q c_{1}^{2}}{2\left(p+q c_{1}^{2}\right)}\right) & =u_{0},
\end{aligned}
$$

$$
\begin{gathered}
u(t)=c_{1} \mathrm{nc}\left(\sqrt{-q c_{1}^{2}-p} t+c_{2} \mid\left(q c_{1}^{2}+2 p\right) / 2\left(q c_{1}^{2}+p\right)\right) \\
u(t)=c_{1} \mathrm{~ns}\left(\left(\sqrt{-q c_{1}^{2}} / \sqrt{2}\right) t+c_{2} \mid-\left(2 p / q c_{1}^{2}\right)-1\right) \\
u(t)=c_{1} \mathrm{nd}\left(\left(\sqrt{-q c_{1}^{2}-2 p} / \sqrt{2}\right) t+c_{2} \mid\left(2\left(q c_{1}^{2}+p\right) /\left(q c_{1}^{2}+2 p\right)\right)\right) \\
u(t)=c_{1} \operatorname{cs}\left(\left(\sqrt{-q c_{1}^{2}} / \sqrt{2}\right) t+c_{2} \mid\left(2\left(q c_{1}^{2}-p\right) / q c_{1}^{2}\right)\right) \\
u(t)=c_{1} \mathrm{ds}\left(\left(\sqrt{-q c_{1}^{2}} / \sqrt{2}\right) t+c_{2} \mid-\left(\left(-q c_{1}^{2}-2 p\right) / 2 q c_{1}^{2}\right)\right) \\
u(t)=c_{1} \operatorname{cd}\left(\left(\sqrt{q c_{1}^{2}+2 p} / \sqrt{2}\right) t+c_{2} \mid-\left(q c_{1}^{2} /\left(q c_{1}^{2}+2 p\right)\right)\right)
\end{gathered}
$$

and the value of $c_{1}$ results from solving the equation $u^{\prime}(0)=\dot{u}_{0}$, i.e.,

$$
-c_{1} \operatorname{sn}\left(c_{2}, m\right) \operatorname{dn}\left(c_{2}, m\right)=\dot{u}_{0} .
$$

Squaring this last equation and taking into account relations (6) and the identities

$$
\begin{aligned}
& \operatorname{dn}^{2}\left(c_{2}, m\right)=1-\operatorname{msn}^{2}\left(c_{2}, m\right)=1-m\left(1-\operatorname{cn}^{2}\left(c_{2}, m\right)\right) \\
& \operatorname{sn}^{2}\left(c_{2}, m\right)=1-\operatorname{cn}^{2}\left(c_{2}, m\right)=1-u_{0}^{2},
\end{aligned}
$$

we arrive at the equation

$$
q c_{1}^{4}+2 p c_{1}^{2}-\left(2 p u_{0}^{2}+q u_{0}^{4}+2 \dot{u}_{0}^{2}\right)=0 .
$$

Solving equation (11) for $c_{1}$ gives

$$
c_{1}= \pm \sqrt{\frac{-p \pm \sqrt{\left(p+q u_{0}^{2}\right)^{2}+2 q \dot{u}_{0}^{2}}}{q}}= \pm \sqrt{\frac{-p \pm \sqrt{\Delta}}{q}} .
$$

To avoid the ambiguity with plus-minus signs, we define

$$
\begin{aligned}
& c_{1}=\sqrt{\frac{\sqrt{\left(p+q u_{0}^{2}\right)^{2}+2 q \dot{u}_{0}^{2}}-p}{q},}, \text { if } \dot{u}_{0}<0, \\
& c_{1}=-\sqrt{\frac{\sqrt{\left(p+q u_{0}^{2}\right)^{2}+2 q \dot{u}_{0}^{2}}-p}{q},} \text { if } \dot{u}_{0} \geq 0, \\
& c_{2}=\mathrm{cn}^{-1\left(\frac{u_{0}}{c_{1}} \frac{q c_{1}^{2}}{2\left(q c_{1}^{2}+p\right)}\right),} \text { if } u_{0} \neq 0, \\
& c_{2}=K\left(\frac{q c_{1}^{2}}{2\left(q c_{1}^{2}+p\right)}\right), \quad \text { if } u_{0}=0 .
\end{aligned}
$$

Making use of the addition formula

$$
\operatorname{cn}(u+v)=\frac{\operatorname{cn}(u) \operatorname{cn}(v)-\operatorname{sn}(u) \operatorname{dn}(u) \operatorname{sn}(v) \operatorname{dn}(v)}{1-\operatorname{msn}^{2}(u) \operatorname{sn}^{2}(v)},
$$

the solution may also be written in the form 


$$
u(t)=\frac{y_{0} \operatorname{cn}(\sqrt{\omega} t \mid m)+\left(\dot{y}_{0} / \sqrt{\omega}\right) \operatorname{sn}(\sqrt{\omega} t \mid m) \operatorname{dn}(\sqrt{\omega} t \mid m)}{1+\operatorname{bsn}(\sqrt{\omega} t \mid m)^{2}}
$$

where

$$
\begin{aligned}
\omega & =\sqrt{\Delta}, \\
m & =\frac{1}{2}\left(1-\frac{p}{\sqrt{\Delta}}\right), \\
b & =\frac{1}{2}\left(\frac{p+q u_{0}^{2}}{\sqrt{\Delta}}-1\right) .
\end{aligned}
$$

The solution is periodic and its main period equals

$$
T=\frac{4 K(m)}{\sqrt{\omega}}, \quad \text { for } 0 \leq m<1 .
$$

In the case, when $m>1$, we make use if the identities

$$
\begin{aligned}
& \operatorname{cn}(t, m)=\operatorname{dn}\left(\sqrt{m} t, \frac{1}{m}\right), \\
& \operatorname{sn}(t, m)=\frac{1}{\sqrt{m}} \operatorname{sn}\left(\sqrt{m} t, \frac{1}{m}\right), \\
& \operatorname{dn}(t, m)=\operatorname{cn}\left(\sqrt{m} t, \frac{1}{m}\right) .
\end{aligned}
$$

The main period will then be

$$
T=\frac{2 K(1 / m)}{\sqrt{m \omega}}, \quad m>0 .
$$

If $m<0$, we transform the solution by means of the following identities:

$$
\begin{aligned}
& \operatorname{cn}(t \mid m)=\operatorname{cd}\left(\sqrt{1-m} t \mid \frac{m}{m-1}\right), \\
& \operatorname{sn}(t \mid m)=\frac{1}{\sqrt{1-m}} \operatorname{sd}\left(\sqrt{1-m} t \mid \frac{m}{m-1}\right), \\
& \operatorname{dn}(t \mid m)=\operatorname{nd}\left(\sqrt{1-m} t \mid \frac{m}{m-1}\right) .
\end{aligned}
$$

Remember that $\mathrm{cd}=\mathrm{cn} / \mathrm{dn}, \mathrm{sd}=\mathrm{sn} / \mathrm{dn}$, and $\mathrm{nd}=1 / \mathrm{dn}$. For reference, Tables 2-4 give useful conversion formulas.

Example 1. Let $p=1, q=1, y_{0}=0$, and $\dot{y}_{0}=2$. The solution to the i.v.p.

$$
u^{\prime \prime}(t)+u(t)+u^{3}(t)=0, \quad u(0)=0, u^{\prime}(0)=2,
$$

is given by

$$
\begin{aligned}
u(t) & =-\frac{2 \sqrt{3} \operatorname{dn}(\sqrt{3} t \mid(1 / 3)) \operatorname{sn}(\sqrt{3} t \mid(1 / 3))}{\operatorname{sn}(\sqrt{3} t \mid(1 / 3))^{2}-3} \\
& =\frac{2 \sqrt{3}}{3} \operatorname{sd}\left(\sqrt{3} t \mid \frac{1}{3}\right) .
\end{aligned}
$$

This solution is periodic with main period $T=4 K(1 / 3) / \sqrt{3}$. See Figure 1 for a comparison with Runge-Kutta numerical solution (dashed curve).

Example 2. Let $p=-5, q=1, y_{0}=1$, and $\dot{y}_{0}=2$. The solution to the i.v.p.,

$$
u^{\prime \prime}(t)-5 u(t)+u^{3}(t)=0, \quad u(0)=1, u^{\prime}(0)=2,
$$

reads

$$
\begin{aligned}
u(t) & =-\sqrt{5+2 \sqrt{6}} \mathrm{cn}\left(2^{3 / 4} \sqrt{[4]} 3 t+\mathrm{cn}^{-1}\left(-\frac{1}{\sqrt{5+2 \sqrt{6}}} \mid \frac{1}{2}+\frac{5}{4 \sqrt{6}}\right) \mid \frac{1}{2}+\frac{5}{4 \sqrt{6}}\right) \\
& =-3.14626 \mathrm{cn}(-2.21336 t-(5.50011-3.13354 \sqrt{-1}) \mid 1.01031) .
\end{aligned}
$$

An equivalent expression without the imaginary unit is

$$
u(t)=\frac{\operatorname{dn}(w t)+2(\sqrt{6}-2) \operatorname{cn}(w t) \mid \operatorname{sn}(w t)}{1-2(\sqrt{6}-2) \operatorname{sn}(w t)^{2}}, \quad \text { being } w=1+\sqrt{\frac{3}{2}}, m=20 \sqrt{6}-48 \approx 0.98979
$$

See Figure 2 for a comparison with Runge-Kutta numerical solution (dashed curve).

$$
\delta=\frac{p^{2}-\Delta}{-q}
$$

it is evident that $\delta>0$. We seek a solution to the i.v.p. (1) in the ansatz form

$$
u(t)=A-\frac{2 A}{1+v(t)},
$$

$$
\delta=2 p u_{0}^{2}+q u_{0}^{4}+2 \dot{u}_{0}^{2} .
$$

Since $\Delta<0$, necessarily $q<0$. From the equality

where $v=v(t)$ is the solution to some Duffing equations 


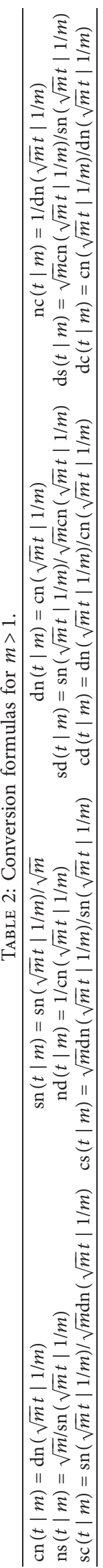




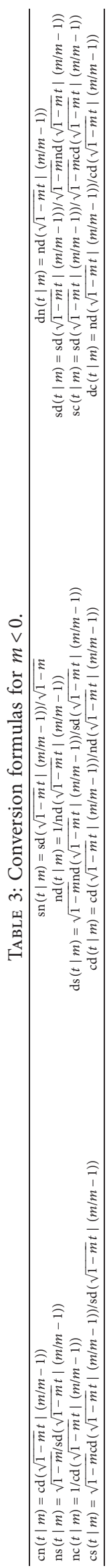




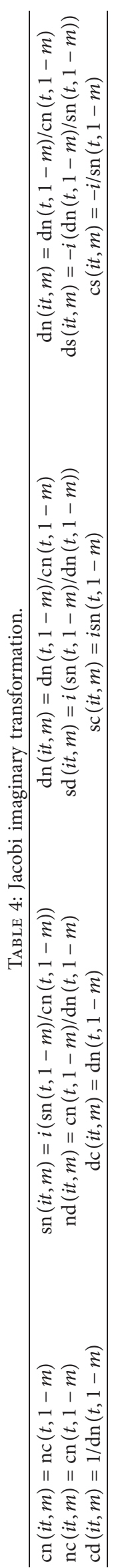




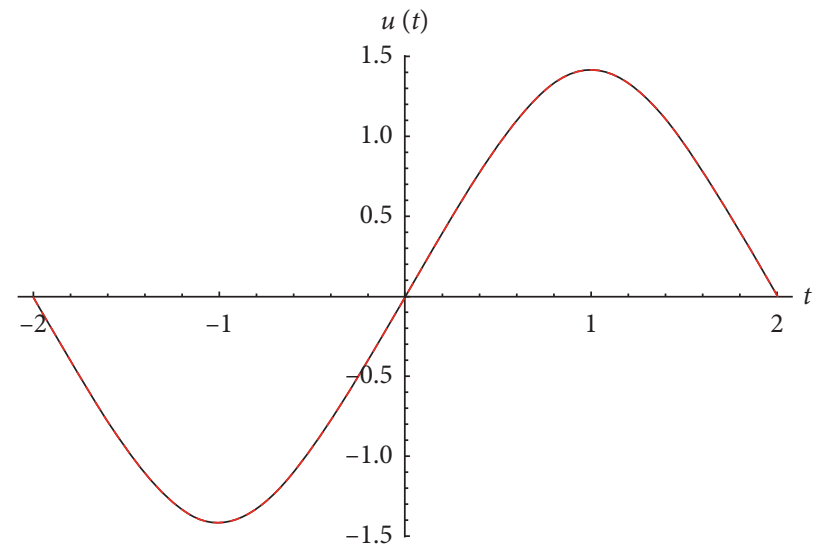

Figure 1: Comparison between the exact and the numerical solution.

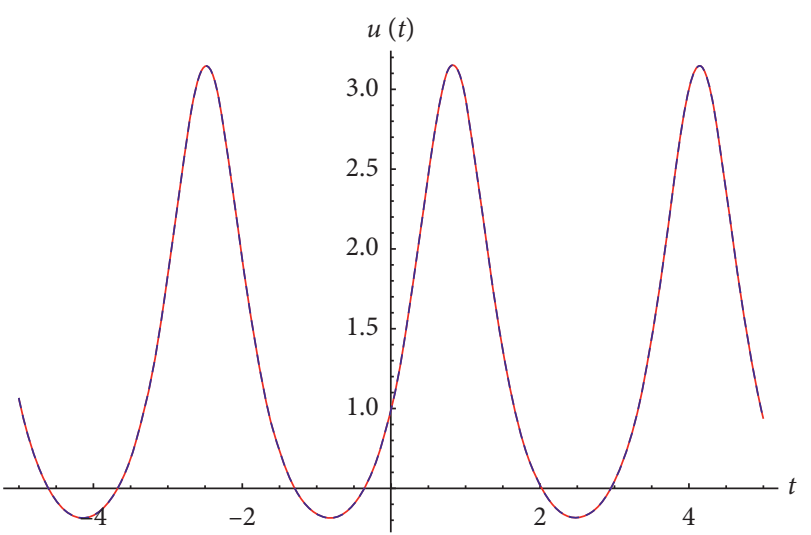

FIgURE 2: Comparison with Runge-Kutta numerical solution (dashed curve).

$$
v^{\prime \prime}(t)+\alpha v(t)+\beta v^{3}(t)=0, \quad v(0)=v_{0}:=\frac{A+u_{0}}{A-u_{0}}, \dot{v}_{0}:=\frac{2 A \dot{u}_{0}}{\left(A-u_{0}\right)^{2}}
$$

We have

$$
\begin{aligned}
v^{\prime \prime}(t) & =-\alpha v(t)-\beta v^{3}(t) \\
v^{\prime}(t)^{2} & =D-\alpha v(t)^{2}-\frac{1}{2} \beta v(t)^{4}, \\
D & =\frac{2 A^{4} \alpha+A^{4} \beta+4 A^{3} u_{0} \beta-4 A^{2} u_{0}^{2} \alpha+6 A^{2} u_{0}^{2} \beta+8 A^{2} \dot{u}_{0}^{2}+4 A u_{0}^{3} \beta+2 u_{0}^{4} \alpha+u_{0}^{4} \beta}{2\left(A-u_{0}\right)^{4}} .
\end{aligned}
$$

Inserting the ansatz (30) into the ode $u^{\prime \prime}(t)+p u(t)+$ $q u^{3}(t)=0$ and taking into account (30) and (31), we get

$$
\begin{aligned}
& u^{\prime \prime}(t)+p u(t)+q u^{3}(t) \\
& \quad=\frac{A}{(1+v(t))^{3}}\left(\left(A^{2} q+p-2 \beta\right) v(t)^{3}+\left(p+2 \alpha-3 A^{2} q\right) v(t)^{2}\right)-\left(p+2 \alpha-3 A^{2} q\right) v(t)-\left(A^{2} q+4 D+p\right)
\end{aligned}
$$


Equating to zero the coefficients of $v^{0}(t), v^{1}(t), v^{1}(t)$, and $v^{3}(t)$ in (32), we obtain an algebraic system. Solving it gives

$$
\begin{aligned}
& \alpha=\frac{1}{2}\left(3 A^{2} q-p\right), \\
& \beta=\frac{1}{2}\left(A^{2} q+p\right), \\
& A=\sqrt[4]{\frac{\delta}{-q}}
\end{aligned}
$$

Observe that

$$
\left(\alpha+v_{0}^{2} \beta\right)^{2}+2 \dot{v}_{0}^{2} \beta=2\left(\sqrt{p^{2}(-q) q \delta}+(-q) \delta\right)>0 .
$$

Thus, the Duffing equation (29) has a positive discriminant. The solution to the i.v.p. (3) is then given by

$$
u(t)=\sqrt[4]{\frac{\delta}{-q}}-\frac{2 \sqrt[4]{\delta /-q}}{1+\left(v_{0} c n(\sqrt{\bar{\omega}} x \mid \bar{m})+\left(\dot{v}_{0} / \sqrt{\omega}\right) \operatorname{sn}(\sqrt{\bar{\omega}} x \mid \bar{m}) d n(\sqrt{\bar{\omega}} x \mid \bar{m})\right) /\left(1+\bar{b} \operatorname{sn}(\sqrt{\bar{\omega}} x \mid \bar{m})^{2}\right)}
$$

where

$$
\begin{aligned}
& \bar{\omega}=\sqrt{\bar{\Delta}}, \\
& \bar{m}=\frac{1}{2}-\frac{\alpha}{2 \sqrt{\bar{\Delta}}}, \\
& \bar{b}=\frac{\alpha+\beta v_{0}^{2}}{2 \sqrt{\bar{\Delta}}}-\frac{1}{2}, \\
& v_{0}=\frac{A+u_{0}}{A-u_{0}}, \\
& \dot{v}_{0}=\frac{2 A \dot{u}_{0}}{\left(A-u_{0}\right)^{2}}, \\
& \bar{\Delta}=\left(\alpha+v_{0}^{2} \beta\right)^{2}+2 \dot{v}_{0}^{2} \beta .
\end{aligned}
$$

The values of $a, \beta$, and $A$ are found from (33).

2.3. Third Case: $\Delta=0$. When the discriminant vanishes, then $q<0$ and the only solution to problem (1) with $u^{\prime}(0)^{2}=$ $\dot{u}_{0}^{2}$ is

$$
u(t)=-\sqrt{-\frac{p}{q}} \tanh \left(\sqrt{\frac{p}{2}} t-\tanh ^{-1}\left(\sqrt{-\frac{q}{p}} u_{0}\right)\right),
$$

which may be verified by direct computation.

\section{New Trigonometric Jacobian Functions}

Define the generalized cosine and sine functions as follows:

$$
\begin{aligned}
& \cos _{\lambda}(t)=\frac{\sqrt{1+\lambda} \cos (\sqrt{1+\lambda} t)}{\sqrt{1+\lambda \cos ^{2}(\sqrt{1+\lambda} t)}}, \\
& \sin _{\lambda}(t)=\frac{\sin (\sqrt{1+\lambda} t)}{\sqrt{1+\lambda \cos ^{2}(\sqrt{1+\lambda} t)}} .
\end{aligned}
$$

Our aim is to find some $\lambda$ so that

$$
\operatorname{cn}(t, m) \approx \cos _{\lambda}(t) \text {. }
$$

Define

$$
R(t)=\frac{1}{2} m x(t)^{4}+\frac{1}{2}(1-2 m) x(t)^{2}+\frac{1}{2} x^{\prime}(t)^{2}+\frac{1}{2}(m-1) .
$$

Observe that $R(t)=0$ when $x(t)=\mathrm{cn}(t, m)$. Let $x(t)=\cos _{\lambda}(t)$. We have

$$
\begin{aligned}
R(t)= & \frac{1}{2\left(\lambda \cos ^{2}(\sqrt{\lambda+1} t)+1\right)^{3}} \\
& \cdot\left(\frac{1}{32}\left(14 \lambda^{2}+2(m+12) \lambda+12 m\right)+\frac{1}{32}\left(-17 \lambda^{2}-32 \lambda-\lambda m-16 m\right) \cos (2 \sqrt{\lambda+1} t)\right. \\
& \left.+\frac{1}{32}\left(2 \lambda^{2}+8 \lambda-2 \lambda m+4 m\right) \cos (4 \sqrt{\lambda+1} t)+\frac{1}{32}\left(\lambda^{2}+\lambda m\right) \cos (6 \sqrt{\lambda+1} t)\right) .
\end{aligned}
$$


We will choose $\lambda$ so that

$$
14 \lambda^{2}+2(m+12) \lambda+12 m=0 .
$$

Define

$$
\lambda=\frac{1}{14}\left(\sqrt{m^{2}-144 m+144}-(m+12)\right) .
$$

The obtained approximations are good. This is seen from Tables 5 and 6.

We now will introduce new Jacobian "trigonometric functions" as follows:

$$
\begin{aligned}
\mathrm{cn}_{m}(t) & =\cos _{\lambda}(t), \quad \text { for }-1 \leq m \leq 0.85, \\
\mathrm{cn}_{m}(t) & =\frac{1}{8}(m-1)(\sinh (2 t)-2 t) \tanh (t) \sec h(t)+\sec h(t), \quad \text { for } 0.85<m \leq 1, \\
\lambda & =\frac{1}{14}\left(\sqrt{m^{2}-144 m+144}-(m+12)\right), \\
\operatorname{sn}_{m}(t) & =\sin _{\lambda}(t), \quad \text { for }-1 \leq m \leq 0.85, \\
\operatorname{sn}_{m}(t) & =\frac{1}{4}(m-1)\left(t \sec h^{2}(t)-\tanh (t)\right)+\tanh (t), \quad \text { for } 0.85<m \leq 1, \\
\lambda & =\frac{1}{14}\left(\sqrt{m^{2}-144 m+144}-(m+12)\right) .
\end{aligned}
$$

Define

$$
\begin{aligned}
\mathrm{dn}_{m}(t) & =\sqrt{1-\mathrm{msn}_{m}^{2}(t)}, \\
\mathrm{nc}_{m}(t) & =\frac{1}{\mathrm{cn}_{m}(t)}, \\
\mathrm{ns}_{m}(t) & =\frac{1}{\mathrm{sn}_{m}(t)}, \\
\mathrm{nd}_{m}(t) & =\frac{1}{\mathrm{dn}_{m}(t)}, \\
\mathrm{sc}_{m}(t) & =\frac{\mathrm{sn}_{m}(t)}{\mathrm{cn}_{m}(t)}, \\
\mathrm{cs}_{m}(t) & =\frac{\mathrm{cn}_{m}(t)}{\mathrm{sn}_{m}(t)}, \\
\operatorname{sd}_{m}(t) & =\frac{\mathrm{sn}_{m}(t)}{\mathrm{dn}_{m}(t)}, \\
\mathrm{ds}_{m}(t) & =\frac{\mathrm{dn}_{m}(t)}{\mathrm{sn}_{m}(t)}, \\
\mathrm{cd}_{m}(t) & =\frac{\mathrm{cn}_{m}(t)}{\mathrm{dn}_{m}(t)}, \\
\mathrm{dc}_{m}(t) & =\frac{\mathrm{dn}_{m}(t)}{\mathrm{cn}_{m}(t)}
\end{aligned}
$$

We extend the new functions (44)-(47) $\mathrm{cn}_{m}(t)$ and $\mathrm{sn}_{m}(t)$ for $m>1$ and $m<0$ and imaginary argument it using Tables 1-3 replacing the $\mathrm{cn}(t, m)$ with $\mathrm{cn}_{m}(t)$ and $\mathrm{cn}_{m}(t)$ with $\mathrm{cn}_{m}(t)$ and so on.

\section{Applications in Physics}

Many partial differential equations arising in soliton theory may be reduced to odes or systems of odes by means of a traveling wave transformation. These odes are generally nonlinear and some of them are Duffing type equations. Let us consider some important models of soliton theory.

4.1. The Klein-Gordon-Zakharov (KGZ) Equation in Plasmas. The KGZ equation reads

$$
\begin{aligned}
q_{t t}-k^{2} q_{x x}+a q+b r q+c|q|^{2} q & =0, \\
r_{t t}-k^{2} r_{x x} & =d\left(|q|^{2}\right)_{x x} .
\end{aligned}
$$

We transform the KGZ by means of the traveling wave substitution

$$
\begin{aligned}
& q(x, t)=u(x-\lambda t) \exp (\sqrt{-1}(-\kappa x+\omega t+\theta)), \\
& r(x, t)=v(x-\lambda t)
\end{aligned}
$$

to obtain the system 
TABLE 5: Errors for approximations (38) and (39).

\begin{tabular}{lcc}
\hline$m$ & $\max _{-T / 2 \leq t \leq T / 2}\left|\operatorname{cn}(t, m)-\cos _{m}(t)\right|$ & $\max _{-T / 2 \leq t \leq T / 2}\left|\operatorname{sn}(t, m)-\sin _{m}(t)\right|$ \\
\hline-1.00 & 0.00672 & 0.00638 \\
-0.95 & 0.00624 & 0.00598 \\
-0.90 & 0.00574 & 0.00557 \\
-0.85 & 0.00526 & 0.00516 \\
-0.80 & 0.00478 & 0.00476 \\
-0.75 & 0.00432 & 0.00435 \\
-0.70 & 0.00385 & 0.00395 \\
-0.65 & 0.00341 & 0.00355 \\
-0.60 & 0.00301 & 0.00316 \\
-0.55 & 0.00261 & 0.00277 \\
-0.50 & 0.00222 & 0.00240 \\
-0.45 & 0.00185 & 0.00203 \\
-0.40 & 0.00151 & 0.00168 \\
-0.35 & 0.00119 & 0.00135 \\
-0.30 & 0.00091 & 0.00104 \\
-0.25 & 0.00065 & 0.00076 \\
-0.20 & 0.00043 & 0.00051 \\
-0.15 & 0.00025 & 0.00030 \\
-0.10 & 0.00011 & 0.00014 \\
\hline
\end{tabular}

TABLe 6: Errors for approximations (38) and (39).

\begin{tabular}{lcc}
\hline$m$ & $\max _{-T / 2 \leq t \leq T / 2}\left|\operatorname{cn}(t, m)-\cos _{m}(t)\right| \max _{-T / 2 \leq t \leq T / 2}\left|\operatorname{sn}(t, m)-\sin _{m}(t)\right|$ \\
\hline 0.00 & 0.00000 & 0.00000 \\
0.05 & 0.00003 & 0.00004 \\
0.10 & 0.00013 & 0.00018 \\
0.15 & 0.00032 & 0.00044 \\
0.20 & 0.00061 & 0.00085 \\
0.25 & 0.00100 & 0.00144 \\
0.30 & 0.00152 & 0.00225 \\
0.35 & 0.00219 & 0.00333 \\
0.40 & 0.00304 & 0.00476 \\
0.45 & 0.00410 & 0.00661 \\
0.50 & 0.00541 & 0.00903 \\
0.55 & 0.00704 & 0.01216 \\
0.60 & 0.00905 & 0.01625 \\
0.65 & 0.01156 & 0.02164 \\
0.70 & 0.01472 & 0.02882 \\
0.75 & 0.01880 & 0.03856 \\
0.80 & 0.02413 & 0.05213 \\
0.90 & 0.03139 & 0.07161 \\
0.95 & 0.04190 & 0.10031 \\
\end{tabular}

$$
\begin{array}{r}
u^{\prime \prime}(\xi)-\frac{u(\xi)\left(a+b v(\xi)+\kappa^{2} k^{2}-\omega^{2}\right)+c u(\xi)^{3}+2 i u^{\prime}(\xi)\left(\kappa k^{2}-\lambda \omega\right)}{(k-\lambda)(k+\lambda)}=0, \quad \xi=x-\lambda t, \\
v^{\prime \prime}(\xi)+\frac{2 d\left(u(\xi) u^{\prime \prime}(\xi)+u^{\prime}(\xi)^{2}\right)}{(k-\lambda)(k+\lambda)}=0, \quad \xi=x-\lambda t .
\end{array}
$$

We choose $\lambda$ so that $\kappa=\left(\lambda \omega / k^{2}\right)$ and integrating the equation (51) twice taking null integration constants, we obtain 


$$
\begin{aligned}
& v(\xi)=\frac{d}{\lambda^{2}-k^{2}} u(\xi)^{2}, \\
& u^{\prime \prime}(\xi)+\frac{1}{k^{2}-\lambda^{2}}\left[a+\omega^{2}\left(\frac{\lambda^{2}}{k^{2}}-1\right)\right] u(\xi)+\frac{c\left(k^{2}-\lambda^{2}\right)-b d}{\left(k^{2}-\lambda^{2}\right)^{2}} u(\xi)^{3}=0,
\end{aligned}
$$

and the problem reduces to solve a Duffing equation.

4.2. The Sine-Gordon Equation. This is the equation

$$
v_{t t}=\alpha v_{x x}+\beta \sin (v) \text {. }
$$

This important model appears in differential geometry and relativistic field theory. It is denominated following its similar form to the Klein-Gordon equation. The equation, as well as several solution techniques, was known in the 19th century, but the equation grew greatly in importance when it was realized that it led to solutions ("kink" and "antikink") with the collision properties of solitons.
The sine-Gordon equation is widely applied in physical and engineering applications, including the propagation of fluxons in Josephson junctions (a junction between two superconductors), the motion of rigid pendular attached to a stretched wire, and dislocations in crystals. It also arises in nonlinear optics. We apply the traveling wave transformation

$$
v(x, t)=2 \arcsin (u(x-\lambda t)),
$$

and the sine-Gordon equation converts into

$$
2\left(\alpha-\lambda^{2}\right)\left(u(\xi)^{2}-1\right) u^{\prime \prime}(\xi)+2 u(\xi)\left(\left(\lambda^{2}-\alpha\right) u^{\prime}(\xi)^{2}-\beta\left(u(\xi)^{2}-1\right)^{2}\right)=0
$$

It may be easily verified that equation (55) holds for any solution $u=u(\xi)$ of the Duffing equation

$$
u^{\prime \prime}(\xi)+\frac{\beta}{\alpha-\lambda^{2}} u(\xi)+\frac{2 \beta}{\lambda^{2}-\beta} u^{3}(\xi)=0 .
$$

4.3. The Pendulum Equation. This equation reads

$$
\begin{aligned}
\theta^{\prime \prime}(t)+\omega^{2} \sin (\theta(t)) & =0, \\
\theta(0) & =\theta_{0}, \\
\theta^{\prime}(0) & =\dot{\theta}_{0} .
\end{aligned}
$$

Let

$$
\theta(t)=2 \arcsin (u(t))
$$

Inserting ansatz (58) into (57) gives

$$
u(t)\left(u^{\prime}(t)^{2}+\omega^{2}\left(u(t)^{2}-1\right)^{2}\right)-\left(u(t)^{2}-1\right) u^{\prime \prime}(t)=0 .
$$

Equation (59) is satisfied for any solution $u=u(t)$ to Duffing equation obeying

$$
\begin{array}{r}
u^{\prime \prime}(t)+\left(p u_{0}^{2}+\frac{1}{2} q u_{0}^{4}+\dot{u}_{0}^{2}+\omega^{2}\right) u(t)-2 \omega^{2} u(t)^{3}=0, \quad u(0)=u_{0}, u^{\prime}(0)=\dot{u}_{0}, \\
\qquad \begin{aligned}
\text { where } u_{0}=u(0)=\sin \left(\frac{\theta_{0}}{2}\right), \dot{u}_{0}=u^{\prime}(0)=\frac{\dot{\theta}_{0}}{2}\left|\cos \left(\frac{\theta_{0}}{2}\right)\right| . \\
\lambda v(\xi)+\frac{a}{2} v(\xi)^{2}+v^{\prime \prime}(\xi)=C,
\end{aligned}
\end{array}
$$

4.4. The KdV Equation. This equation originated from soliton theory. It reads

$$
\partial_{t} u+\partial_{x x x} u+a u \partial_{x} u=0, a=\text { non zero constant. }
$$

Let $u=v(x+\lambda t)=u(\xi)$. The traveling wave substitution gives $\lambda v^{\prime}(\xi)+a v(\xi) v^{\prime}(\xi)+v^{\prime \prime \prime}(\xi)=0$. Integrating once, we obtain where $C$ is the constant of integration. We seek a solution to the nonlinear ode (62) in the ansatz form

$$
v(\xi)=R+S y^{2}(\xi), \quad \text { where } y^{\prime \prime}(\xi)+p y(\xi)+q y^{3}(\xi)=0,
$$


where the constants $p, q, R$, and $S$ are to be determined. Since $\int y^{\prime}(\xi)\left(y^{\prime \prime}(\xi)+p y(\xi)+q y^{3}(\xi)\right) \mathrm{d} \xi=\int 0 \mathrm{~d} \xi=D$, it is clear that

$$
y^{\prime}(\xi)^{2}=D-p y(\xi)^{2}-\frac{q}{2 y(\xi)^{4}}
$$

Inserting the ansatz (63) into (62) and taking into account (63), we obtain

$$
\left(\frac{a S^{2}}{2}-3 q S\right) y(\xi)^{4}+(a R S-4 p S+\lambda S) y(\xi)^{2}+\frac{a R^{2}}{2}+C+2 D S+\lambda R=0
$$

Equating the coefficients of $y(\xi)^{4}, y(\xi)^{2}$, and $C+2 D S+$ $\lambda R$ to zero gives an algebraic system. Solving it, we arrive at the expressions

$$
\begin{aligned}
& p=\frac{1}{4}(a R+\lambda), \\
& q=\frac{a S}{6}, \\
& S \neq 0 .
\end{aligned}
$$

4.5. The Nonlinear Schrodinger Equation. The nonlinear Schrödinger equation is among the most prominent equations in nonlinear physics, especially in nonlinear optics. The nonlinear Schrödinger equation is of particular importance in the description of nonlinear effects in optical fibers. The nonlinear Schrödinger equation is a central model of nonlinear science, applying to hydrodynamics, plasma physics, molecular biology, and optics. It has been studied for more than 40 years, and it is employed in numerous fields well beyond plasma physics and nonlinear optics, where it originally appeared. The nonlinear Schrödinger equation (NLSE) is in the following form:

$$
\sqrt{-1} \frac{\partial u}{\partial t}+\frac{\partial^{2} u}{\partial x^{2}}+\gamma|u|^{2} u=0
$$

where $\gamma$ is a nonzero real constants and $u=u(x, t)$ is a complex valued function of two real variables $x, t$. The Schrödinger equations occur in various areas of physics, including nonlinear optics, plasma physics, superconductivity, and quantum mechanics. The NLSE (67) exhibits soliton and periodic cnoidal wave solutions. Let

$$
u(t, x)=e^{i(\alpha x+\beta t)} v(x-2 \alpha t) .
$$

Under this transformation, the NLSE (67) takes the form

$$
v^{\prime \prime}(\xi)-\left(\alpha^{2}+\beta\right) v(\xi)+2 \gamma v^{3}(\xi)=0,
$$

which is a Duffing equation.

\section{Data Availability}

No data were used to support this study.

\section{Conflicts of Interest}

The authors declare that they have no conflicts of interest.

\section{References}

[1] G. Duffing, Erzwungene schwingungen bei veränderlicher eigenfrequenz und ihre technische bedeutung, Vieweg \& Sohn, Braunschweig, Germany, Series: Sammlung Vieweg, 1918.

[2] Kovacic and M. J. Brennan, The Duffing Equation: Nonlinear Oscillators and Their Behavior, John Wiley \& Sons, Hoboken, NJ, USA, 1st edition, 2011.

[3] A. A. Tsonis, Chaos from Theory to Applications, Springer, Berlin. Germany, 1992.

[4] G. C. Layek, An Introduction to Dynamical Systems and Chaos, Springer, Berlin, Germany, 2015.

[5] Z. Feng, G. Chen, G. Chen, and S.-B. Hsu, "A qualitative study of the damped duffing equation and applications," Discrete \& Continuous Dynamical Systems-B, vol. 6, no. 5, pp. 1097-1112, 2006.

[6] K. Johannessen, "An analytical solution to the equation of motion for the damped nonlinear pendulum," European Journal of Physics, vol. 35, Article ID 035014, 2014.

[7] K. Johannessen, "The Duffing oscillator with damping," European Journal of Physics, vol. 36, Article ID 065020, 2015.

[8] S. A. El-Tantawy, A. H. Salas, and E. C. H Jairo, E. Jairo, Stability analysis and novel solutions to the generalized degasperis procesi equation: an application to plasma physics," PLoS One, vol. 16, no. 9, Article ID e0254816, 2021.

[9] A. H. S. Salas, "Analytic solution to the generalized complex Duffing equation and its application in soliton theory," Applicable Analysis, vol. 100, no. 13, pp. 2867-2872, 2019.

[10] A. H. Salas, "Analytic solution to the pendulum equation for a given initial conditions," Journal of King Saud University Science, vol. 32, no. 1, pp. 974-978, 2020.

[11] A. H. Salas and J. E. Castillo, "Exact solutions to cubic Duffing equation for a nonlinear electrical circuit," Visión electrónica, vol. 8, 2014.

[12] A. H. Salas and J. E. Castillo, "Exact solution to duffing equation and the pendulum equation," Applied Mathematical Sciences, vol. 8, pp. 8781-8789, 2014.

[13] A. H. Salas, J. E. Castillo, and D. J. Mosquera, "A new approach for solving the undamped helmholtz oscillator for the given arbitrary initial conditions and its physical applications," Mathematical Problems in Engineering, vol. 2020, Article ID 7876413, 7 pages, 2020.

[14] A. H. Salas and S. A. El-Tantawy, "On the approximate solutions to a damped harmonic oscillator with higher-order nonlinearities and its application to plasma physics: semianalytical solution and moving boundary method," The 
European Physical Journal Plus, vol. 135, no. 10, pp. 833-917, 2020.

[15] A. H. Salas, S. A. El Tantawy, and J. E. Castillo, "The hybrid finite difference and moving boundary methods for solving a linear damped nonlinear schrödinger equation to model rogue waves and breathers in plasma physics," Mathematical Problems in Engineering, vol. 2020, Article ID 6874870, 11 pages, 2020.

[16] A. H. Salas and S. A. El Tantawy, "New method for solving strong conservative odd parity nonlinear oscillators: applications to plasma physics and rigid rotator," AIP Advances, vol. 10, no. 8, Article ID 085001, 2020.

[17] A. H. Salas, S. A. El Tantawy, and A. A. Youseff, "New solutions for chirped optical solitons related to kaup-newell equation: application to plasma physics," Optik, vol. 218, Article ID 165203, 2020.

[18] H. Alvaro and T. Casanova, "A new approach for solving the complex cubic-quintic duffing oscillator equation for given arbitrary initial conditions," Mathematical Problems in Engineering, vol. 2020, Article ID 3985975, 8 pages, 2020. 